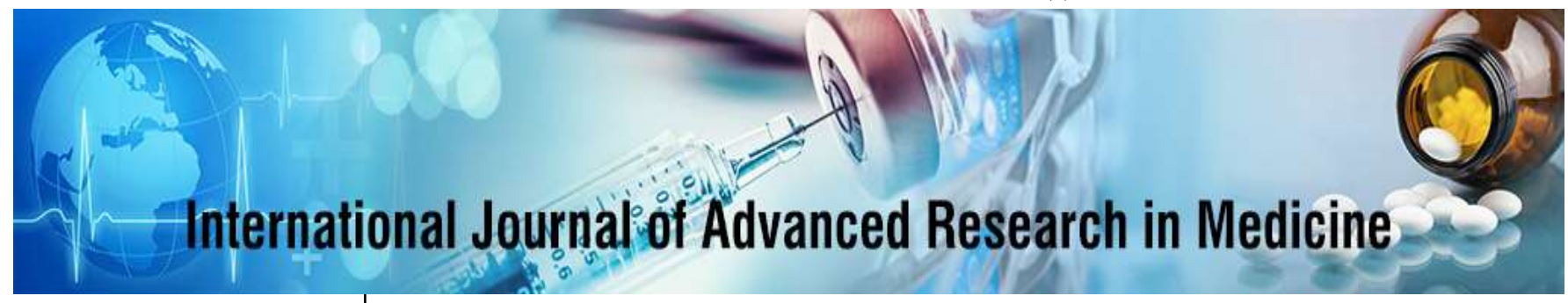

E-ISSN: 2706-9575 P-ISSN: 2706-9567 IJARM 2021; 3(2): 150-153 Received: 18-05-2021 Accepted: 21-06-2021

\section{Dr. Samina Tarannum} Associate Professor, Department of General Medicine, Osmania Medical College, Hyderabad, Telangana, India
Corresponding Author: Dr. Samina Tarannum Associate Professor, Department of General Medicine, Osmania Medical College, Hyderabad, Telangana, India

\title{
Correlation between serum vitamin-d and essential hypertension
}

\section{Dr. Samina Tarannum}

DOI: $\underline{\text { https://doi.org/10.22271/27069567.2021.v3.i2c.234 }}$

\begin{abstract}
Background: Inadequate vitamin D affects about 50\% of the world's population. Hypovitaminosis D pandemic may largely be attributed to lifestyles and environmental conditions which limit exposure to sun. Adequate exposure to sunlight is necessary for synthesis of ultraviolet-B (UVB) - induced vitamin-D in the skin.
\end{abstract}

Objective: To study the correlation between the level of vitamin- D and the presence of essential hypertension in patients presenting to a hospital.

Methods: The subjects were segregated into two groups: Group 1 (case group) - 50 patients with essential hypertension, Group 2 (control group) - 50 ostensibly healthy individuals. Each subject was interviewed and a standardized questionnaire was answered and completed, which included demographics, anthropometric profile, individual characteristics associated with major risk factors for cardiovascular disease, past medical history, sun exposure details (type of job and average time spent in the sun per day), and biochemical parameters. Hypertension was documented based on disclosure of known hypertensive patients self-reporting on out-patient visit or newly diagnosed hypertensives based on physician measurements. Vitamin D levels were measured from a venous sample taken at the time of hospital visit.

Results: The control group comprised 50 patients, 34 of whom were male and 16 of whom were female, and 50 hypertensive patients, 36 of whom were male and 14 of whom were female. There was no significant difference in the gender distribution of the study and control groups $(\mathrm{P}=0.8272)$. The hypertensive group had an age of $58.7 \pm 9.3$ years and the normotensive group $60.5 \pm 5.3$ years. There was no statistically significant difference. The normotensive and hypertensive patients studied had no significant differences in height, weight, or BMI $(p>0.05)$. The normotensive group's systolic and diastolic blood pressures $(114 \pm 5.89$ and $75.0 \pm 5.0)$ were considerably lower than the hypertensive group's (162.10 \pm 16.5 and $95.90 \pm 9.30)$.

Conclusion: Vitamin D and essential hypertension have an inverse relationship. When systolic and diastolic blood pressure were considered as continuous variables, a substantial relationship of high blood pressures corelating with low vitamin-D levels was observed. In addition, left ventricular hypertrophy was also shown to be correlated to hypovitaminosis D.

Keywords: Vitamin-D, Hypertension, Calcium, Hypovitaminosis D

\section{Introduction}

The deficiency in vitamin D is quite widespread and of paramount significance, especially with regard to Indian public health. Vitamin D insufficiency is known to be related to several of the musculoskeletal disorders. Vitamin D supplementation is advised in order to avoid rickets in children and osteomalacia in adults. Vitamin $\mathrm{D}$ controls bone and mineral metabolism and hemostasis. It has been found that all human cells have vitamin D receptors (VDRs). Hence vitamin D insufficiency has been associated with a number of chronic extra skeletal illnesses ${ }^{[1]}$.

Inadequate vitamin D levels affects about $50 \%$ of the world's population. Hypovitaminosis D pandemic may largely be attributed to lifestyles and environmental conditions which limit exposure to sun which is necessary for skin synthesis of ultraviolet-B (UVB) - induced vitamin-D ${ }^{[2]}$.

Black people absorb more UVB than white people due to difference in skin's melanin and consequently need more sunlight in order to create equal quantities of vitamin $\mathrm{D}^{[3]}$. During winter months, levels of UVB radiation decrease with an increased distance from earth's equator. Similarly, in areas with increased air pollution, UV-B rays reach the earth surface inadequately. 
Consequently, increases in blood pressure levels were all associated with different variables causing lower UVBinduced vitamin D production, such as high latitude, industrialization, and dark skin ${ }^{[4]}$.

Deficiency of vitamin D has an additional component of elevated risk of cardiovascular events, although it remains uncertain if this combination has a direct correlation. One of the probable reasons for a correlation between vitamin D and cardiovascular diseases could be the influence of vitamin D on blood pressure ${ }^{[5]}$.

In this study, we will outline the processes hypothesized to underpin the association between vitamin D and systemic arterial hypertension, as well as analyze the clinical data supporting this relationship.

\section{Materials and methods}

Study Design: It's a Cross-Sectional Study

Sample Size: 100 patients (50 Cases and 50 Controls)

\section{Inclusion Criteria}

- Patients over the age of 45 years with essential hypertension.

Exclusion Criteria: Patients with

- $\quad$ 45 years of age

- Calcium/Vitamin - D supplementations

- Long term diuretics

- Pregnancy

- Secondary hypertension

- Other comorbid conditions like
- diabetes mellitus

- hypothyroidism

- renal failure

Controls: Age and gender matched participants above the age of 45, attending out-patient clinics of departments of Ophthalmology and Otorhinolaryngology with no history of systemic hypertension and having normal blood pressure, and who fulfilled the aforementioned exclusion criteria.

The study group determined by the aforementioned criteria (inclusion and exclusion criteria) was first briefed about the study's purpose. After obtaining written informed consent from willing participants, the subjects were enrolled. The subjects were segregated into two groups: Group 1 (patient group) - 50 patients with essential hypertension, Group 2 (control group) - 50 ostensibly healthy individuals. Each subject was interviewed and a standardized questionnaire was filled up, which included demographics, anthropometric profile, individual characteristics associated with major risk factors for cardiovascular disease, past medical history, sun exposure details (type of job and average time spent in the sun per day), and biochemical parameters. On the baseline and follow-up assessments, hypertension was detected based on self-reports or physician measurements.

Statistical Analysis: All data were statistically evaluated using the t-test and the chi-square test in SPSS Version 21, with $p<0.05$ deemed significant.

\section{Observation and results}

Table 1: Distribution based on age and gender across cases and controls

\begin{tabular}{|c|c|c|c|c|}
\hline \multirow{2}{*}{ Sex } & \multicolumn{2}{|c|}{ Normotensive cases } & \multicolumn{2}{|c|}{ Hypertensive Cases } \\
\hline & No & $\%$ & No & $\%$ \\
\hline Male & 34 & $68 \%$ & 36 & $72 \%$ \\
\hline Female & 16 & $32 \%$ & 14 & $28 \%$ \\
\hline Total & 50 & $100 \%$ & 50 & $100 \%$ \\
\hline P-Value & \multicolumn{4}{|c|}{0.8272 Not significant } \\
\hline \multicolumn{5}{|l|}{ AGE GROUP (in years) } \\
\hline $45-50$ & 10 & $20 \%$ & 18 & $36 \%$ \\
\hline $51-60$ & 18 & $36 \%$ & 12 & $24 \%$ \\
\hline $61-70$ & 21 & $42 \%$ & 16 & $32 \%$ \\
\hline$>70$ & 1 & $2 \%$ & 4 & $8 \%$ \\
\hline Total & 50 & $100 \%$ & 50 & $100 \%$ \\
\hline Mean \pm S.D & \multicolumn{2}{|c|}{$60.5+5.3$} & \multicolumn{2}{|c|}{$58.7 \pm 9.3$} \\
\hline P-Value & \multicolumn{4}{|c|}{0.131} \\
\hline Variables & Mean & SD & Mean & SD \\
\hline Height ( in cms) & 166.5 & 6.0 & 163.5 & 6.5 \\
\hline P-Value & \multicolumn{4}{|c|}{0.1110} \\
\hline Weight ( in kgs) & 70.5 & 7.5 & 66.1 & 8.5 \\
\hline P-Value & \multicolumn{4}{|c|}{0.1049} \\
\hline BMI & 25.41 & 2.79 & 25.5 & 3.05 \\
\hline P-Value & \multicolumn{4}{|c|}{0.669} \\
\hline SBP & 114 & 5.89 & 162.10 & 16.5 \\
\hline P-Value & \multicolumn{4}{|c|}{0.0001} \\
\hline DBP & 75.0 & 5.0 & 95.90 & 9.30 \\
\hline P-Value & \multicolumn{4}{|c|}{0.0001} \\
\hline
\end{tabular}

The control group comprised 50 patients, 34 of whom were male and 16 of whom were female, and 50 hypertensive patients, 36 of whom were male and 14 of whom were female. There was no significant difference in the gender distribution of the study and control groups $(\mathrm{P}=0.8272)$.
The hypertensive group had an age of $58.7 \pm 9.3$ years and the normotensive group $60.5 \pm 5.3$ years. There was no statistically significant difference.

The normotensive and hypertensive patients studied had no significant differences in height, weight, or BMI $(p>0.05)$. 
The normotensive group's systolic and diastolic blood pressures $(114 \pm 5.89$ and 75.0 \pm 5.0$)$ were considerably lower than the hypertensive group's $(162.10 \pm 16.5$ and 95.90土9.30).

Table 2: Distribution based on Vitamin-D level across Cases and controls

\begin{tabular}{|c|c|c|c|c|}
\hline \multirow{2}{*}{ Vitamin D level } & \multicolumn{2}{|c|}{ Normotensive Cases } & \multicolumn{2}{|c|}{ Hypertensive Cases } \\
\hline & No & $\%$ & No & $\%$ \\
\hline Level - I - $\left(<37.5 \mathrm{nmol} \mathrm{L-}^{-1}\right)$ & 3 & $6.0 \%$ & 29 & $58.0 \%$ \\
\hline Level II - $\left(<37.5-49.9 \mathrm{nmol} \mathrm{L-}^{1}\right)$ & 10 & $20.0 \%$ & 4 & $8.0 \%$ \\
\hline Level III - $\left(50-74.9 \mathrm{nmol} \mathrm{L}^{-1}\right)$ & 27 & $54.0 \%$ & 10 & $20.0 \%$ \\
\hline Level IV- $\left(75-100 \mathrm{nmol} \mathrm{L}^{-1}\right)$ & 10 & $20.0 \%$ & 7 & $14.0 \%$ \\
\hline Vit D ( in nmol L-1) Range & \multicolumn{2}{|c|}{$24.9-90$} & \multicolumn{2}{|c|}{$20.8-88.9$} \\
\hline Mean+S.D & \multicolumn{2}{|c|}{$62.4 \pm 18.5$} & \multicolumn{2}{|c|}{$46.5 \pm 20.5$} \\
\hline P-Value & \multicolumn{4}{|c|}{0.0071 Significant } \\
\hline
\end{tabular}

The normotensive patients had a Vitamin D level of 62.4 $\mathrm{nmol} / \mathrm{L}$ on an average. This was substantially greater than the mean values observed in hypertensive patients (46.5 nmol L-1). This was a statistically significant.

Table 3: Distribution based on Vitamin - D levels and other parameters

\begin{tabular}{|c|c|c|c|c|c|}
\hline \multirow{2}{*}{ Variable } & \multicolumn{4}{|c|}{ Value (Mean \pm SD) for cases with Vit. D level } & \multirow{2}{*}{ ' $\mathbf{p}$} \\
\cline { 2 - 5 } & I & II & III & IV & $\begin{array}{c}0.6670 \\
\text { Not significant }\end{array}$ \\
\hline Urea & $27.7 \pm 7.3$ & $23.3 \pm 1.6$ & $28.3 \pm 7.5$ & $29 \pm 6.9$ & $\begin{array}{c}0.0059 \\
\text { Significant }\end{array}$ \\
\hline Corr. Calcium & $7.65 \pm 0.41$ & $7.58 \pm 0.45$ & $8.02 \pm 0.30$ & $8.5 \pm 0.50$ & $\begin{array}{c}0.5439 \\
\text { Not significant }\end{array}$ \\
\hline Phosphate & $3.97 \pm 0.69$ & $3.8 \pm 1.30$ & $3.86 \pm 0.79$ & $3.52 \pm 0.49$ & $\begin{array}{c}0.4623 \\
\text { Not significant }\end{array}$ \\
\hline Sr. Creatinine & $1.2 \pm 0.25$ & $1.07 \pm 0.19$ & $1.16 \pm 0.15$ & $1.13 \pm 0.15$ & $\begin{array}{c}0.7159 \\
\text { Not significant }\end{array}$ \\
\hline
\end{tabular}

In hypertensives, there was a positive correlation between calcium and vitamin D levels.

Table 4: Vitamin-D levels and ECG Changes

\begin{tabular}{|c|c|c|c|c|c|c|c|c|c|}
\hline \multirow{2}{*}{ Risk factors - ECG } & \multicolumn{7}{|c|}{ No. of cases with Vit D level } & \multirow{3}{*}{ P-Value } \\
\cline { 2 - 11 } & \multicolumn{2}{|c|}{ I } & \multicolumn{3}{|c|}{ II } & \multicolumn{3}{|c|}{ III } & \multicolumn{2}{|c|}{ IV } & \\
\cline { 2 - 11 } & No & \% & No & \% & No & \% & No & \% & \\
\hline LAD, LVH (36) & 30 & 83.3 & 1 & 2.7 & 3 & 8.3 & 2 & 5.5 & 0.0001 \\
\hline WNL (14) & 2 & 14.2 & 3 & 21.4 & 5 & 35.7 & 4 & 28.5 & Significant \\
\hline
\end{tabular}

In instances where vitamin D levels were low, the percentage of ECG abnormalities was substantially greater ( level I)

Table 5: Vitamin-D and concentric LVH

\begin{tabular}{|c|c|c|c|c|c|c|c|c|c|}
\hline \multirow{3}{*}{ Risk factors - Echo } & \multicolumn{8}{|c|}{ No. of cases with Vit D level } & \multirow{3}{*}{ P-Value } \\
\hline & \multicolumn{2}{|c|}{ I } & \multicolumn{2}{|c|}{ II } & \multicolumn{2}{|c|}{ III } & \multicolumn{2}{|c|}{ IV } & \\
\hline & No & $\%$ & No & $\%$ & No & $\%$ & No & $\%$ & \\
\hline Normal (32) & 7 & 21.8 & 5 & 15.6 & 11 & 34.3 & 9 & 28.1 & 0.0001 \\
\hline Abnormal (18) & 16 & 88.8 & 2 & 11.1 & - & - & & - & Significant \\
\hline
\end{tabular}

The percentage of aberrant echocardiographs was substantially greater in cases when vitamin D levels were low (level I)

Table 6: Vitamin D levels and BP

\begin{tabular}{|c|c|c|c|c|}
\hline \multirow{2}{*}{ Vit D level } & \multicolumn{2}{|c|}{ SBP } & \multicolumn{2}{c|}{ DBP } \\
\cline { 2 - 5 } & Mean & SD & Mean & SD \\
\hline I & 175.23 & 9.8 & 101.3 & 8.3 \\
\hline II & 154.23 & 3.6 & 96.3 & 5.3 \\
\hline III & 146 & 3.9 & 88.65 & 4.2 \\
\hline IV & 141 & 2.7 & 84.38 & 4.5 \\
\hline P-Value & \multicolumn{4}{|c}{$(0.0001)$ significant } \\
\hline
\end{tabular}

There was a significant relationship between blood pressure values and vitamin $\mathrm{D}$ levels in hypertension patients ( $\mathrm{p}$ $0.05)$.

\section{Discussion}

Hypertension in both developed and developing nations is a significant cause of mortality and morbidity, which is 
generally diagnosed by health care workers during regular checks.

The correlation between vitamin D deficiency and high blood pressure can be attributed to a number of mechanisms. Even though the link between circulating vitamin $\mathrm{D}$ levels and renin activity has previously been highlighted in important clinical hypertension research, $1.25(\mathrm{OH}) 2-\mathrm{D}$ has only recently been shown to directly influence the renin-angiotensin system.

Other possible pathways include vitamin D's effects on vessel wall, cells such as endothelial cells, vascular smooth muscle cells, and macrophages, which all express the vitamin D receptor (VDR) and $1 \alpha$-hydroxylase. As a result, a normal level of BP is considered to need an optimum amount of circulating $1,25(\mathrm{OH}) \mathrm{D}$, which is regulated by $25(\mathrm{OH}) \mathrm{D}$ concentrations.

Our findings corroborate these processes, and Burgaz et al. found that males with vitamin D levels of $<37.5 \mathrm{nmol} / \mathrm{L}$ had a three-fold higher risk of hypertension compared to those with normal levels (>75 nmol/L) ${ }^{[6,7]}$.

In this study age, gender, and physical characteristics such as BMI were all adjusted between the normotensive and hypertension groups. The ' $p$ ' value indicated that there was no confounding factor. Random blood sugar (RBS) and renal functions also were matched for both groups. Conversely, as compared to normotensive individuals, serum uric acid was found to be higher in the hypertension group. However, relation of increased uric acid levels and vitamin D levels has to be determined. Cannon et al. did a study that showed similar results of increased uric acid levels in hypertensive individuals ${ }^{[8]}$.

In contrast to the normotensive group, hypertensive patients had retinopathy, left ventricular hypertrophy on echocardiography, and electrocardiographic abnormalities suggesting left axis deviation and left ventricular hypertrophy. The mean vitamin-D level in male hypertension patients was lower than that in females, although the difference was not statistically significant. This is in accordance to research by Bansal et al and his colleagues ${ }^{[9]}$.

When compared to hypertensive patients, the serum vitamin-D level was substantially lower in normotensive patients. Hintzpeter B et al., found a substantial negative relationship between plasma $25(\mathrm{OH}) \mathrm{D}$ levels and blood pressure and hypertension in his observational research ${ }^{[10]}$. Although numerous research by Burgaz et al., and other authors have shown an inverse relationship between vitamin-D and hypertension, few studies, such as the one by Formann et al., have shown no significant relationship between vitamin-D level and hypertension ${ }^{[11]}$. Given the uncertainties, it is recommended that prospective studies be conducted among vitamin-D deficient individuals and followed up on to determine the rate of incident hypertension.

\section{Conclusion}

Vitamin D and essential hypertension have an inverse relationship. Left ventricular hypertrophy was shown to be correlated to hypovitaminosis D. When systolic and diastolic blood pressure were considered as continuous variables, a substantial relationship with low vitamin-D levels was observed. Hence regular measurement and subsequent replacement of vitamin D must be recommended as a regular practice.

\section{References}

1. Holick MF. Vitamin D deficiency. N. Engl. J. Med 2007;357:266-281.

2. Rostand SG. Ultraviolet light may contribute to geographic and racial blood pressure differences. Hypertension 1997;30:150-156.

3. Krause R, Bühring M, Hopfenmüller W, Holick MF, Sharma AM. Ultraviolet B and blood pressure. Lancet 1998;352:709-710.

4. Melamed ML, Michos ED, Post W, Astor B. 25hydroxyvitamin D levels and the risk of mortality in the general population. Arch. Intern. Med. 2008;168:16291637.

5. Autier P, Gandini S. Vitamin D supplementation and total mortality: a meta- analysis of randomized controlled trials. Arch. Intern. Med 2007;167:17301737.

6. Burgaz A, Byberg L, Rautiainen S, Orsini N, Håkansson N, Arnlöv J, et al. Confirmed hypertension and plasma $25(\mathrm{OH}) \mathrm{D}$ concentrations amongst elderly men. J Intern Med 2011;269(2):211-8. Doi: 10.1111/j.1365-2796.2010.02309.x. Epub 2010 Nov 23. PMID: 21091810.

7. Anderson JL, May HT, Horne BD, et al. Relation of vitamin $\mathrm{D}$ deficiency to cardiovascular risk factors, disease status, and incident events in a general healthcare population. Am J Cardiol 2010;106:963-8

8. Cannon PJ, stason WB, demartini FE, sommers SC, laragh JH. Hyperuricemia in primary and renal hypertension. N Engl j Med 1966; 275:457-464.

9. Bansal N, Katz R, Appel L, Denburg M, Feldman H, Go AS, et al. CRIC Study Investigators. Vitamin D Metabolic Ratio and Risks of Death and CKD Progression. Kidney Int Rep 2019;4(11):1598-1607. Doi: 10.1016/j.ekir.2019.08.014. PMID: 31891001; PMCID: PMC6933450.

10. Hintzpeter B, Mensink GB, Thierfelder W, Muller MJ, Scheidt-Nave C. Vitamin D status and health correlates among German adults. Eur J Clin Nutr 2008;62:10791089.

11. Forman JP, Curhan GC, Taylor EN. Plasma 25hydroxyvitamin D levels and risk of incident hypertension among young women. Hypertension 2008;52:828-32. 